\title{
Severe asthma phenotyping: the applicability of current proposols in daily practice
}

\begin{abstract}
Background: Severe asthma (SA) is also need to be phenotyping. We aimed to demonstrate the applicability of current definition and classification proposals in daily practice.
\end{abstract}

Methods: SA patients, according to WHO definition of treatment resistant SA, were enrolled from the allergy clinic. The SARP algorith define and WHO proposal were applied to the subjects independently by asthma experts. Demographic and clinical characteristics of the subjects were used for the comparison.

Results: Ninety-five (F/M: 68/27) patients with a mean age of $45.97 \pm 9.44$ years (range25-71) were enrolled. The patients were grouped as controlled treatmentresistant SA (56\%) and uncontrolled treatment resistant SA group (44\%). There was no statistically significant difference between the groups but aspirin hypersensitivity. $49 \%$ of patients was classified as exacerbates, $26 \%$ as fixed airway obstruction group and $23 \%$ as undefined (other $2 \%$ ). Age and mold sensitivity was higher; FEV1/FVC and FEF25-75 were lower in exacerbation-prone SA. More than half of the patients were define clustered at 4 and 5 according to SARP proposol. Patients with fixed obstruction were only in the clusters 4 and 5 .

Conclusion: Current phenotyping proposols failed to cover all SA patients. Thus, there is still need further investigations in order to explore validity and applicability of the phenotyping of SA.

Keywords: adult asthma, severe asthma, phenotype, cluster analysis, refractory asthma, difficult-to-treat asthma
Volume I Issue I - 2014

\author{
Adile Berna Dursun, ${ }^{1,2}$ Ferda Oner Erkekol, ${ }^{2}$ \\ Ozlem Kar Kurt ${ }^{2,3}$ \\ 'Department of Internal Medicine, Division of Immunology and \\ Allergic Diseases, Recep Tayyip Erdogan University School of \\ Medicine, Turkey \\ ${ }^{2}$ Divison of Immunology and Allergic Diseases, Ataturk Chest \\ Disease and Thoracic Surgery Trainig and Research Hospital, \\ Turkey \\ ${ }^{3}$ Department of Respiratory Medicine, Izzet Baysal University \\ School of Medicine, Turkey
}

\begin{abstract}
Correspondence: Adile Berna Dursun, Department of Internal Medicine, Division of Immunology and Allergic Diseases, Recep Tayyip Erdogan University School of Medicine, Rize, Turkey, Tel 905325874868, Email aberna.dursun@gmail.com
\end{abstract}

Received: August 12, 2014 | Published: August 21, 2014

\section{Introduction}

Asthma affects 300million people of all ages in the world and the prevalence of asthma is increasing. ${ }^{1}$ Fortunately, most patients with asthma have a mild-to-moderate disease and can be controlled with optimal treatment. Around $10 \%$ of the overall asthmatics suffer from ongoing symptoms, frequent exacerbations and reduced quality of life despite appropriate therapy. The cost of illness related to asthma is extremely high, and more than $50 \%$ of the asthma patient care costs are attributed to asthma cases with ongoing symptoms due to unscheduled health care utilization, hospitalizations and the cost of pharmacotherapy. ${ }^{2,3}$ Thus, in recent years, scientific attention mostly has been focused on the severe asthma (SA).

Various definitions have emerged to characterize the clinical features of SA, but essential feature of this phenotype is the need for high doses of inhaled corticosteroids plus long-acting bronchodilators to achieve disease control. ${ }^{4}$ European Respiratory Society defined the term difficult asthma in 1999, and subsequently American Thoracic Society defined the term refractory asthma. Later an ERS workshop statement on SA has defined the disease as being reserved to those patients who have refractory asthma despite extensive reevaluation over a 6-month period. In 2009, the World Health Organization (WHO) proposed a global definition of SA. The new classification identifies three broad categories of SA: untreated SA, difficult-to-treat SA and treatment-resistant SA. ${ }^{5}$

Several multicenter cohort studies of SA patients have been published. The ENFUMOSA study was a multicenter European observational study comparing 163 subjects with SA and 158 patients with asthma controlled on low-dose ICS and demonstrated that SA may be characterized by small airway diseases ${ }^{6}$ as is all RAD. The TENOR study, including 4756 patients from 283 sites, sought to establish characteristics of patients with very poorly controlled asthma over a 2year observational period in the United States and identified allergic sensitization, post bronchodilator lung function, the use of nonprivate health care insurance, and treatment by a pulmonologist as significant and independent predictors of having consistently very poor controlled asthma over a 2 year period. ${ }^{7}$ Another US multicenter cohort study (SARP) establish that SA patients were older, had a longer duration of disease, and had more frequent emergency health care utilization. ${ }^{8}$ The UK Severe Asthma Network (382 patients from 4 centers) has identified the importance of intercenter variation in clinical characteristics such as more preserved lung function in Asian ethnicity and greater allergic sensitization to house dust mite in London than the other centers. ${ }^{9}$ In other words, both European and North American severe asthma cohorts strongly support the presence of different subgroups or phenotypes. ${ }^{10}$

Phenotyping of SA becomes an essential element of the new approaches for personalized management. Despite significant interest demonstrated by specialists, most physicians are unable to fully understand the clustering process, and its translation in the real world to treat asthma is not clear. ${ }^{11}$ In this study, the main purpose was classified SA patients according to WHO definition, and the secondary aim was to compare phenotypes by using both clinical presentation and SARP clustering system in order to observe the manifestation of daily clinical practice. 


\section{Materials and methods}

\section{Study population}

In this retrospective study, 95 patients with a diagnosis of treatment-resistant SA according to WHO universal definitions were enrolled at the Department of Allergic Diseases at our center between 2006 and 2011. Patients under 18years and/or with lung disease other than asthma were excluded. Patients were consecutively collected from subjects admitted to the department due to uncontrolled asthma despite being prescribed high levels of asthma medications. Before the enrollment, all patients were reevaluated by asthma experts certified in both pulmonology and allergic diseases. A standardized questionnaire based on demographic characteristics, asthma control and severity, and medication use was administered to all participants. For the initial visit, detailed personal and familial history, physical examination, diagnostic tests, chest radiography, spirometry with bronchodilator test, allergy skin testing, lab tests (IgE, peripheral eosinophil count), assessing inhaler technique, triggers and comorbidities were evaluated. Follow-up visits were performed with 4-6weeks intervals at least for 6 months. When necessary, computerized tomography scans of the chest were taken and consulted for comorbidities with related disciplines: ENT, polysomnography, gastroenterology, psychiatry, radiology, interventional bronchology.

\section{Pulmonary function tests}

Spirometry was performed by trained respiratory technicians using a flow-sensor spirometer (Sensormedics, Vmax 229, Yorba Linda-California). Pre-bronchodilator spirometry was performed for baseline \% predicted FEV1. We confirmed the patients had withheld medication appropriately prior to testing. Post-bronchodilator spirometry was obtained after salbutamol $(400 \mathrm{mcg})$ administration and was defined as maximal \% predicted FEV1. Reversibility was defined as a $12 \%$ change in FEV1.

\section{Evaluation of atopy}

Atopy was defined as a positive skin test to at least one of the aeroallergens. Glycerinated extracts of house dust mites, cockroach, molds (aspergillus, alternaira, cladosporium, penicllium), cat and dog epithelia, grass, tree and weed pollen antigens were used (Stallergenes, Antony/France). The puncture method with a $1 \mathrm{~mm}$ tip disposable lancet was used, and a wheal reaction with a mean diameter of $3 \mathrm{~mm}$ greater than the negative control was accepted as a positive response.

\section{Phenotyping}

All patients were in the group 3 (treatment-resistant SA) of WHO severe asthma definition. Later, patients were assigned to the two subgroups. Group A consisted of patients in which control could be maintained, group B consisted of patients in which control was not achieved. ${ }^{5}$ Secondarily, patients were clustered using three variables (baseline FEV1-with a bronchodilator withhold-, maximal FEV1 after six-to eight puffs of albuterol, and age of onset of asthma) as in SARP. ${ }^{8}$

\section{Statistical analysis}

The Statistical Package for Social Sciences (SPSS v.17; Chicago, IL, USA) was used to analyze the data. Numeric values were expressed as mean $\pm \mathrm{SD}$ and categorical/ordinal values as $\mathrm{n}(\%)$ and results were expressed as the mean $(95 \%$ confidence interval $) \pm$ SEM. Nonparametric tests were used due to heterogeneity of the groups. Differences between study groups were examined by Kruskal Wallis test, and any significant differences were further analyzed by pairwise comparisons using Mann-Whitney U test. All directional $p$ values were two-tailed, and significance was assigned to values lower than 0.05 .

\section{Results}

A total of 95 patients, 68 female (71.6\%) and 27 male (28.4\%), with a mean age of $45.97 \pm 9.44$ years (25-71) were enrolled. 61(64.2\%) patients had never smoked, and nearly $45 \%$ of all patients were obese. 33(34.7\%) patients had aspirin hypersensitivity proved by ASA provocation test, $4(4.29 \%)$ have other drug hypersensitivities. 46(48.4\%) patients had nasal polyps. 56(62.1\%) were classified as non-atopic. The mean time to diagnosis was $11.41 \pm 7.9$ (1-33years). The mean baseline FEV1 level was $1.45 \pm 0.53(0.45-3.01) \mathrm{L}$ and $49.43 \pm 13.9(21-83) \%$ predicted. $47(49.5 \%)$ patients were taking more than 3 drugs. Of the 95 patients, 45 patients were hospitalized more than twice per year. The demographic and clinical details of the study population are summarized in Table 1.

Table I Demographics and clinical characteristics of study population $(n=95)$

\begin{tabular}{|c|c|}
\hline Sex, n (\%) & \\
\hline Female & $68(71.6 \%)$ \\
\hline Male & $27(28.4 \%)$ \\
\hline \multicolumn{2}{|l|}{ Age } \\
\hline Mean $\pm \mathrm{SD}$ (min-max), years & $45.97 \pm 9.44(25-71)$ \\
\hline \multicolumn{2}{|l|}{ Asthma duration } \\
\hline Mean $\pm \mathrm{SD}($ min-max), years & $11.41 \pm 7.9(\mid-33)$ \\
\hline \multicolumn{2}{|l|}{ Smoking Status, n (\%) } \\
\hline Never smoker & $61(64.2 \%)$ \\
\hline Ex-smoker & $29(30.5 \%)$ \\
\hline Active smoker & $5(5.39 \%)$ \\
\hline \multicolumn{2}{|l|}{ Drug Allergy, n (\%) } \\
\hline None & $58(61.1 \%)$ \\
\hline ASA hypersensitivity & $33(34.7 \%)$ \\
\hline Others(antibiotics, RCM) & $4(4.29 \%)$ \\
\hline \multicolumn{2}{|l|}{ Nasal Polyp, n (\%) } \\
\hline No & $49(51.6 \%)$ \\
\hline Yes & $46(48.4 \%)$ \\
\hline \multicolumn{2}{|l|}{ Obesity Status, n (\%) } \\
\hline Normal & $20(21.1 \%)$ \\
\hline Overweight & $3 I(32.6 \%)$ \\
\hline Obese & $40(42.1 \%)$ \\
\hline Morbid obese & $4(4.2 \%)$ \\
\hline \multicolumn{2}{|l|}{ Atopy Status, n (\%) } \\
\hline Nonatopic & $56(62.1 \%)$ \\
\hline Atopic & $36(37.9 \%)$ \\
\hline House dust mites sensitivity & $27(75 \%)$ \\
\hline Moulds sensitivity & $12(33.3 \%)$ \\
\hline Cat sensitivity & $7(19.4 \%)$ \\
\hline Cockroach sensitivity & $3(8.3 \%)$ \\
\hline Pollen sensitivity & $12(33.3 \%)$ \\
\hline \multicolumn{2}{|l|}{ Baseline FEVI } \\
\hline Mean $\pm S D$ (min-max), L & $1.45 \pm 0.53(0.45-3.01)$ \\
\hline Mean $\pm S D(\min -\max ), \%$ predicted & $49.43 \pm 13.9(2 \mid-83)$ \\
\hline
\end{tabular}


Table Continued..

\begin{tabular}{ll}
\hline \multicolumn{2}{l}{ Baseline FEVI } \\
\hline Medications used, $\mathrm{n}(\%)$ & \\
ICS & $95(100 \%)$ \\
LABA & $95(95 \%)$ \\
LTRA & $89(93.7 \%)$ \\
Theophylline & $27(28.4 \%)$ \\
Oral CS & $6(6.3 \%)$ \\
Anti lgE & $9(9.5 \%)$ \\
ASA desensitization & $17(17.9 \%)$ \\
$\neq$ of Medicaitons used, $\mathbf{n}(\%)$ & \\
3 & $48(50.5 \%)$ \\
$>3$ & $47(49.5 \%)$ \\
$\neq$ of Hospitalization for Asthma, & \\
$\mathbf{n}(\%)$ & \\
0 & $27(28.4 \%)$ \\
I & $23(24.2 \%)$ \\
$\geq 2$ & $45(47.4 \%)$ \\
\hline
\end{tabular}

The study group was clustered as follows: $56 \%$ of the patients were in the group A (controlled treatment-resistant SA), 44\% of the patients were in group B (uncontrolled treatment-resistant SA). There was no significant difference between two groups according to age, gender, age at diagnosis, smoking status, obesity, nasal polyposis, atopy, parameters of PFT or the number of drugs used. Aspirin hypersensitivity was significantly higher in group B (Group A/Group B: 30.3\%/69.7\%) ( $\mathrm{p}=0.048)$ (Graphic 1).

According to clinical phenotypes, the patients were divided into three groups. $49 \%$ of patients had frequent exacerbations, $26 \%$ of patients had irreversible obstruction according to FEV1 and 23\% of undefined or did not have these 2 criteria? There was no significant difference between groups with respect to gender, age at diagnosis, cigarette smoke, obesity, drug hypersensitivity, atopy, the amount of drugs used. Age was significantly higher in exacerbation-prone severe asthma group. Analysis of lung function revealed that FEV1/ FVC and FEF25-75 were also lowest in this group. Mold sensitivity was higher in the exacerbation-prone severe asthma group. Aspirin hypersensitivity was higher in the undefined group (Table 2). $63 \%$ of exacerbation-prone SA patients were in treatment resistant asthma group B according to WHO.

Using the SARP algorithm, SA patients were clustered as follows. $2.1 \%$ of subjects $(n=2)$ are grouped into cluster $1.31 .6(\%)(n=30)$ of subjects are grouped in Cluster 2. 10.5\% $(\mathrm{n}=10)$ of subjects are grouped in Cluster 3. Cluster 4 was the largest group $(n=32 ; 33.6 \%)$. The remaining $22.1 \%(\mathrm{n}=21)$ of patients assigned into cluster 5 . The patients who had fixed obstruction was only at clusters 4 and 5 .

Table 2 Comparison of features of SA patients according to clinical presentation

\begin{tabular}{|c|c|c|c|}
\hline Age*,y & $45.63 \pm 9.26$ & $51.12 \pm 9.35$ & $41.25 \pm 7.29$ \\
\hline FEVI $\min L^{\epsilon}$ & $1.45 \pm 0.46$ & $1.14 \pm 0.37$ & $\mathrm{I} .77 \pm 0.6 \mathrm{I}$ \\
\hline FEVI min \%" & $50.13 \pm 14.45$ & $43.04 \pm 9.61$ & $55.00 \pm 14.76$ \\
\hline FEF25-75 $\min ^{\infty}$ & $30.46 \pm 15.07$ & $20.7 \pm 7.52$ & $32.63 \pm 16.39$ \\
\hline Mold sensitization, $\mathrm{n}$ & 11 & $\mathrm{I}$ & - \\
\hline ASA tolerant, $n$ & 33 & 17 & 8 \\
\hline ASA intolerant ${ }^{*}, \mathrm{n}$ & II & 7 & 15 \\
\hline
\end{tabular}

*Exa. vs fixed 0.05

Exa. vs unclassified 0.031

Fixed vs unclassified $<0.00$ I

EExa. vs fixed 0.004

Exa. vs unclassified 0.049

Fixed vs unclassified $<0.00$

\#Exa. vs fixed 0.04

Exa. vs unclassified $>0.05$

Fixed vs unclassified 0.004

$\infty$ Exa. vs fixed 0.007

Exa. vs unclassified 0.007

Fixed vs unclassified $<0.00$ I

$¥$ Unclassified vs exa. and fixed 0.04

\section{Discussion}

In the current study, according to WHO clinical phenotyping system $23 \%$ of the SA patients were not able to be classified. Using the SARP clustering system, $1 / 3$ of the patients clustered in group 2 with normal lung functions and more than half of the patients were clustered in group 4 and 5 with female predominance, decreased lung functions and non-atopic status. Thus two different classification schemes for SA failed to cover all SA in daily clinical practice.

Asthma, and SA in particular, is increasingly recognized as a heterogeneous disease. Many definitions have been used for
$\mathrm{SA}^{3,7,8}$ and also many previous studies have attempted to define phenotypes of severe asthma. ${ }^{5,6,9}$ Phenotypes may be divided into 2 main categories such as clinical phenotypes and inflammatory phenotypes, ${ }^{10}$ According to clinical features the 3 most relevant clinical phenotypes are exacerbation prone SA, SA with fixed airflow obstruction and oralcorticosteroid-dependent SA. ${ }^{11}$ Patients with frequent exacerbations are susceptible to exacerbations as a result of modifiable and nonmodifiable risk factors. ${ }^{12}$ Fixed obstruction is probably related to bronchial inflammation, which worsens after exacerbations and airway remodeling. ${ }^{13}$ Childhood-onset, female gender, smoking and genetic predisposition are the most important risk factors contributing to developing fixed obstruction phenotype. 
Several possible mechanisms have been suggested to explain corticosteroid-dependent SA. ${ }^{14}$ The inflammatory phenotypes were defined according to inflammatory markers and cells by using noninvasive methods such as induced sputum, peripheral blood, urine, exhaled NO and electronic noses. ${ }^{15}$ Three inflammatory phenotypes have been described; persistent eosinophilic SA, noneosinophilic SA with increased neutrophils and paucigranulocytic SA. Several studies have demonstrated eosinophilic or noneosinophilic inflammation in asthma by using pathobiologic characteristics to guide asthma management. ${ }^{16,17}$ But some of these features may not always obtainable in clinical settings.

It is more important to classify asthma according to clinical presentation, pathophysiologic characteristics, and response to treatment. These features include lung function level, age of onset, triggering factors, frequency and severity of exacerbations, comorbidities, site of inflammation, remodeling changes and airway smooth muscle dysfunction. The Severe Asthma Research Program (SARP) performed cluster analysis of severe persistent asthma patients using 34 variables and defined five clusters. They also developed a simplified SARP algorithm for sorting based on three variables available in the clinical setting (baseline percent of predicted FEV1, maximal \% predicted FEV1, age of asthma onset). ${ }^{6}$

Recently, 6 asthma endotypes have been demonstrated of which at least 4 are relevant to severe asthma. These 4 endotypes are early-onset severe allergic asthma, late-onset persistent eosinophilic asthma, aspirin exacerbated airway diseases and allergic bronchopulmonary mycoses. ${ }^{18}$ Similarly (in this context), Amelink et al also confirmed that obese adult-onset asthma phenotype is not associated with eosinophilic airway inflammation and responsiveness to ICS but is characterized by poor asthma control, low quality of life and high health care utilization. ${ }^{19}$ In the current study, we used cluster analysis to identify five clusters of SA and we compared the phenotyping according to clinical features (irreversible obstruction and exacerbation-prone severe asthma). Cluster 1 was the smallest $(2,1 \%)$ group, differing from SARP. In clusters 1 and 2 , half of the patients were atopic and had normal lung function. Cluster 2 subjects had early-onset asthma. Cluster 3 was a group of older, obese, mostly female subjects who were shown to have mild airway obstruction. This group was similar to that described in SARP and with the group of obese subjects with late-onset asthma. ${ }^{6}$ Cluster 4 was the largest group and was predominantly female, non atopic, decreased lung function and early onset. Cluster 5 was late onset, non atopic, predominantly female and lowest lung function. These findings are similar to results of Amelink et al. ${ }^{19}$

Two unexpected results of the study were lower atopy rates and more female predominanc than those of the previous studies. ${ }^{6,9}$ The most attractive finding of the study is that the majority of ASA hypersensitive patients were classified as neither exacerbation-prone nor fixed airway obstruction group. Thus, it may suggest that ASA hypersensitive SA is distinct clinical phenotype. Another striking result was that being elderly and having mold sensitivity, unrelated to obesity and gender, were found to be distinctive features of exacerbation-prone phenotype.

Although the present study was designed and conducted in tertiary allergy clinic by experienced asthma experts, it still has some limitations. First, the number of subjects is small. Second, we did not use path biological features for end typing due to the retrospective nature of the study.
In conclusion, the present study shows that actual classification/ phenotyping/clustering proposals for severe asthma will miss characterizing some individuals in this group of patients. It is well known that asthma is heterogeneous disease and even SA comprises various clinical and path physiological phenotypes. Improved characterization of the disease could contribute to improved selection of appropriate treatment and a greater understanding of its path physiology and natural history. The results of ongoing studies such as "Unbiased Biomarkers for the Prediction of Respiratory Diseases" Outcome (U-BIOPRED) consortium should contribute to more detailed phenotyping of severe asthama. ${ }^{14}$

\section{Acknowledgements}

None.

\section{Conflict of interest}

The author declares no conflict of interest.

\section{References}

\section{1. www.ginasthma.org}

2. Wenzel S. Severe asthma in adults. Am J Respir Crit Care Med. 2005;172(2):149-160.

3. Chanez P, Wenzel SE, Anderson GP, et al. Severe asthma in adults: what are the important questions? J Allergy Clin Immunol. 207;119(6):13371348 .

4. Bell MC, Busse WW. Severe asthma: An expanding and mounting clinical challenge. J Allergy Clin Immunol Pract. 2013;1(2):110-121.

5. Siddiqui S, Gonem S, Wardlaw AJ. Advances in the management of severe asthma. Semin Respir Crit Care Med. 2012;33(6):666-684.

6. The ENFUMOSA cross-sectional European multicentre study of the clinical phenotype of chronic severe asthma. European Network for Understanding Mechanisms of Severe Asthma. Eur Respir J. 2003;22(3):470-477.

7. Dolan CM, Fraher KE, Bleecker ER, et al. Design and baseline characteristics of the epidemiology and natural history of asthma: outcomes and treatment regimens (TENOR) study: a large cohort of patients with severe or difficult-to-treat asthma. Ann Allergy Asthma Immunol. 2004;92(1):32-39.

8. Moore WC, Bleecker ER, Curran-Everett D, et al. Characterization of the severe asthma phenotype by the National Heart, lung, blood institute's severe asthma research program. J Allergy Clin Immunol. 2007;119(2):405-413.

9. Heaney LG, Brightling CE, Menzies-Gow A, et al. Refractory asthma in the UK: cross-sectional findings from a UK multicenter registry. Tho$\operatorname{rax}$. 2010;65(9):787-794.

10. Gaga M, Zervas E, Gibson PG. Allergic and non-allergic factors in severe asthma. Eur Resp Mon. 2011;51:107-219.

11. Bel EH, Sousa A, Fleming L, et al. Diagnosis and definition of severe refractory asthma: an international consensus statement from the Innovative Medicine Initiative (IMI). Thorax. 2011;66(10):910-917.

12. Miller MK, Lee JH, Miller DP, et al. Recent asthma exacerbations: a key predictor of future exacerbations. Respir Med. 2007;101(3):481-489.

13. Bel EH. Clinical phenotypes of asthma. Curr Opin Pulm Med. 2004;10(1):44-50.

14. Campo P, Rodriguez F, Sanchez-Garcia S, et al. Phenotypes and endotypes of uncontrolled severe asthma: new treatments. J Investig Allergol Clin Immunol. 2013;23(2):76-88. 
15. Gibson PG, Wang F, He XY, et al. Noninvasive assessment of inflammation in severe asthma. Eur Resp Mon. 2011;51:208-217.

16. Wenzel S. Severe asthma: from characteristics to phenotypes to endotypes. Clin Exp Allergy. 2012;42(5):650-8.

17. Bourdin A, Chanez P. Clustering in asthma: why, how and for how long? Eur Respir J. 2013;41(6):1247-1248.
18. Young Y. Phenotypes and endotypes of severe asthma in children. Korean J Pediatr. 2013;56(5):191-195

19. Amelink M, de Nijs SB, de Groot JC, et al. Three phenotypes of adult onset asthma. Allergy. 2013;68(5):674-80. 